\title{
A CERTAIN TYPE OF LOCALLY COMPACT TOTALLY DISCONNECTED TOPOLOGICAL GROUPS ${ }^{1}$
}

\author{
T. S. WU
}

Let $G$ be a locally compact totally disconnected topological group. If $G$ contains a closed subgroup $A$ which is isomorphic with a discrete finitely generated free abelian group, such that $G / A$ is compact then we shall call $G$ a totally disconnected two end group. In this note, we shall give a structure theorem of such groups.

Notations. $P(G)$ denotes the subset of $G$ consisting of all compact elements of $G$; i.e. $g \in P(G)$ if and only if the closure of the subgroup generated by $g$ is compact. $B(G)$ denotes the (characteristic) subgroup of $G$ consisting of all the elements whose orbits under the inner automorphisms is relative compact, i.e. $g \in B(G)$ if and only if $\left\{f g f^{-1} \mid f \in G\right\}$ has compact closure.

LEMMA 1 (UsAKov [2]). Let $G$ be a topological group containing a compact open subgroup $H$. Let $B$ be a periodic invariant subset of $G$ with compact closure (periodic means that every element in $B$ is a compact element in $G$ ). Then the closure of the subgroup generated by $\bar{B}$ is a compact normal subgroup of $G$.

Lemma 2. If $G$ is a totally disconnected locally compact group, then $P(G) \cap B(G)$ is a characteristic subgroup of $G$.

Proof. For $z \in G$, let $O(z)=\left\{g z g^{-1} \mid g \in G\right\}$. Suppose $x, y \in P(G)$ $\cap B(G)$. Then $O(x) \cup O(y) \subset P(G) \cap B(G)$, and $O(x) \cup O(y)$ has compact closure; moreover it is an invariant subset of $G$. By Lemma 1 $\operatorname{cl}(O(x) \cup O(y))$ generates a subgroup whose closure is compact, a fortiori, $x y \in P(G) \cap B(G)$, and $P(G) \cap B(G)$ is a subgroup of $G$. It is characteristic, since $P(G)$ and $B(G)$ are characteristic subgroups of $G$.

Theorem. Suppose $G$ is a locally compact totally disconnected topological group containing a closed discrete subgroup $A$ such that

(1) $A$ is a finitely generated free abelian group,

(2) $G / A$ is compact.

Then there exists a compact characteristic subgroup $K$ of $G$ such that

(1) $G / \mathrm{cl}(B(G))$ is compact,

(2) $\operatorname{cl}(B(G)) / K$ is a discrete $F C$ group; i.e. each conjugacy class in this group is a finite set.

Received by the editors November 4, 1968.

1 This research was supported in part by NSF Grants GP-7527 and g-GP-8961. 
Proof. It is easy to see that $A \subset B(G) \subset \operatorname{cl}(B(G))$. Thus $\operatorname{cl}(B(G))$ is compact.

Let $F$ denote $\operatorname{cl}(B(G))$. Then $\operatorname{cl}(B(F))=F, A \subset F$ and $F / A$ is compact. By Lemma 2, $B(F) \cap P(F)$ is a characteristic subgroup of $F$. Given any compact open subgroup $L$ of $F, B(F) \cap L \subset B(F) \cap P(F)$, and $B(F) \cap L$ is dense in $L$. Hence $\operatorname{cl}(B(F) \cap P(F))$ is an open subgroup of $F$. Since $B(F) \cap P(F) \cap A=\{e\}$, $e$ identity; also $P(F) \cap B(F)$ is open (hence closed) in $B(F)$, hence $\operatorname{cl}(P(F) \cap B(F)) \cap B(F)$ $=P(F) \cap B(F)$. This implies $\operatorname{cl}(P(F) \cap B(F)) \cap A=\{e\}$. Because $F / A$ is compact, $F$ is $\sigma$-compact. Then $\operatorname{cl}(P(F) \cap B(F)) A$ is an open (hence closed) subgroup of $F$, and $\operatorname{cl}(P(F) \cap B(F)) A / A$ is a closed subset of the compact space $F / A$ and $\operatorname{cl}(P(F) \cap B(F))$ is compact. Let $K$ $=\operatorname{cl}(P(F) \cap B(F))$. Then $F / K$ is discrete. It is now clear that $B(F / K)$ $=F / K$, and the conjugacy class of any element in $F / K$ is a finite set. This completes our proof.

REMARK. In fact, all we really need in the proof of the above theorem is: $G$ contains a closed subgroup $A$ with the properties:

(1) $G / A$ is compact,

(2) $A \subset B(G)$, and

(3) $A \cap P(G)=\{e\}$.

REMARK. In general, $G / A$ is compact does not imply that $\operatorname{cl}(B(G))$ $=G$. For example, group generated by two elements $a, b$ subjected to the relation $a^{2}=b^{2}=e$.

\section{REFERENCES}

1. B. H. Neumann, Groups with finite classes of conjugate elements, Proc. London Math. Soc. 1 (1951), 178-187.

2. V. I. Usakov, Classes of conjugate elements in topological groups, Ukrain. Mat. Z. 14 (1962), 366-371.

Case Western Reserve University 